\title{
Array-Based Beamforming to the Vertebral Canal: Demonstration of Feasibility
}

\author{
Rui Xu \\ Department of Medical Biophysics \\ University of Toronto \\ Toronto, Canada \\ ORCID:ruixu
}

\author{
David Martin \\ Department of Medical Biophysics \\ University of Toronto \\ Toronto, Canada \\ dave.martin@mail.utoronto.ca
}

\author{
Meaghan A. O'Reilly \\ Physical Sciences \\ Sunnybrook Research Institute \\ Toronto, Canada \\ moreilly@sri.utoronto.ca
}

\begin{abstract}
Focused ultrasound blood-spinal cord barrier opening is a promising new method for improving the non-invasive delivery of therapeutic agents to the spinal cord. A custom 64-element phased array was built, based on the design of a component of a spine-specific array for focusing ultrasound through vertebral laminae and correct vertebra-induced distortions. Geometric and hydrophone delays were tested in each thoracic vertebra in an ex vivo spine $(n=12)$. Pressure fields were measured in each canal to measure focal aberrations. Geometric delays produced foci shifted by $2.4 \pm 1.7 \mathrm{~mm}$ from the target, while hydrophone delays produced foci $1.3 \pm 1.1 \mathrm{~mm}$ from the target. Simulation showed good correlation with hydrophone delays and may be a viable non-invasive method for phase corrections. This work demonstrates the feasibility of transvertebral ultrasound beamforming with a phased-array.
\end{abstract}

Index Terms-Focused Ultrasound, Spine, Vertebral Canal, Phased Array

\section{INTRODUCTION}

Focused ultrasound blood-spinal cord barrier opening has been used in preclinical models to improve the non-invasive delivery of therapeutic agents to the spinal cord [1, 2]. Controlling an ultrasound focus in the human vertebral canal requires the convergence of imaging, acoustic modeling and computation, and phased array technology [3]. We previously used simulation to design a spine-specific phased array that merges the need to propagate through bone, as is necessary in transskull applications, with the availability of acoustic windows between the vertebrae, as are found in the rib cage [4, 5]. Here, we demonstrate the transvertebral beamforming ability of one 64-element array modified from the design a quadrant of the 256-element spine-specific array [5]. Two simulation methods were tested; the multi-layered ray acoustics model in [4], and the k-Space pseudospectral fluid model in the opensource k-Wave Matlab toolbox [6].

\section{Methods}

\section{A. Device Construction}

The 64-element array was constructed using cylindrical zirconate titanate (PZT-4) elements with outer diameters of

This research was funded by NSERC (National Science and Engineering Research Council of Canada), the Ontario Ministry of Research, Innovation and Science, the Canada Foundation for Innovation John Evans Leaders Fund/Ontario Research Fund - Research Infrastructure, and the Canada Research Chair Program.
$7.5 \mathrm{~mm}$, inner diameters of $5.3 \mathrm{~mm}$, and heights of $4.5 \mathrm{~mm}$ (DeL Piezo Specialties, LLC). The elements were integrated into a 64-module 3D printed element housing, designed based on the optimized design for a 64-element translaminar array component [5]. Some modifications were made during design process: the elements were placed flat within the cylindrical surface of the printed housing, giving elements that were cylindrically focused to the vertical axis, and the element spacing was increased by $1.75 \mathrm{~mm}$ to create space for the press-fit housings. The array cylindrical radius of curvature was doubled to $24 \mathrm{~cm}$. 24 AWG tinned copper lead wire (Arcor) was threaded through openings in the element housings and silver epoxied (GPC-251LV, Creative Materials) to the element; one ground on the inner surface of the element, one signal on the outer surface of the element, to drive the elements at their length mode resonance frequency. Marine Epoxy (LePage) was used to solidify the silver epoxy connections. A PZT-4 epoxy mixture (3:1 ratio) was used as backing material for each element to optimize the impulse response of the elements and allow the generation of short pulses. A parylene coating was applied to the assembly. Microcoaxial cables were soldered to the lead wires through the parylene coating, leading to an ITT Cannon DL5-260RW6B connected to a custom printed circuit board (PCBUnlimited) with element-specific matching circuits. Each element was individually matched at $400 \mathrm{kHz}$ to $50 \Omega$ impedance, $0^{\circ}$ phase. The PCB was connected via a second ITT canon to a Verasonics Vantage ${ }^{\mathrm{TM}} 256$ Low Frequency System with the Extended Transmit Option.

\section{B. Experimental Setup}

Thoracic vertebrae from an ex vivo adult human spine (Osta International, White Rock, BC, Canada) were submersed in a $10 \%$ buffered formalin solution for storage and to maintain their acoustic properties. Each vertebra was degassed in deionized water in a vacuum chamber (Nalgene vacuum chamber, Fisher Scientific; Gas, Benton Harbor, MI, USA) for two hours prior to use in experiment. Each vertebra was imaged with a clinical CT scanner (Aquilone One, Toshiba) at approximately $0.5 \mathrm{~mm}$ resolution for CT-based beamforming simulations. All experiments were performed in a rubberlined tank (dimensions: $x=90 \mathrm{~cm}, y=30 \mathrm{~cm}, z=30 \mathrm{~cm}$ ) mounted on an optical table. An electronic 3-axis positioning 
system (VelMex) was mounted on the optical table, and defined the coordinate system $(x, y, z)$ of the experiment. A needle hydrophone (Precision Acoustics, $0.5 \mathrm{~mm}$ diameter) was mounted to the Velmex arm, with the needle aligned with $z$ (vertical) axis. The hydrophone was connected to an oscilloscope (Tektronix MDO3014) that digitized the signals and sent them to the Verasonics Workstation. The 64-element array was mounted at the $+x$ end of the tank, with the mean element propagation path aligned with the $x$-axis. Vertebrae were individually mounted in the tank on a manual 3-axis translation stage. The vertebrae were positioned approximately $12 \mathrm{~cm}$ from the array, along the array x-axis. A diagram and photos depicting the experimental setup is shown in Fig. 1 .

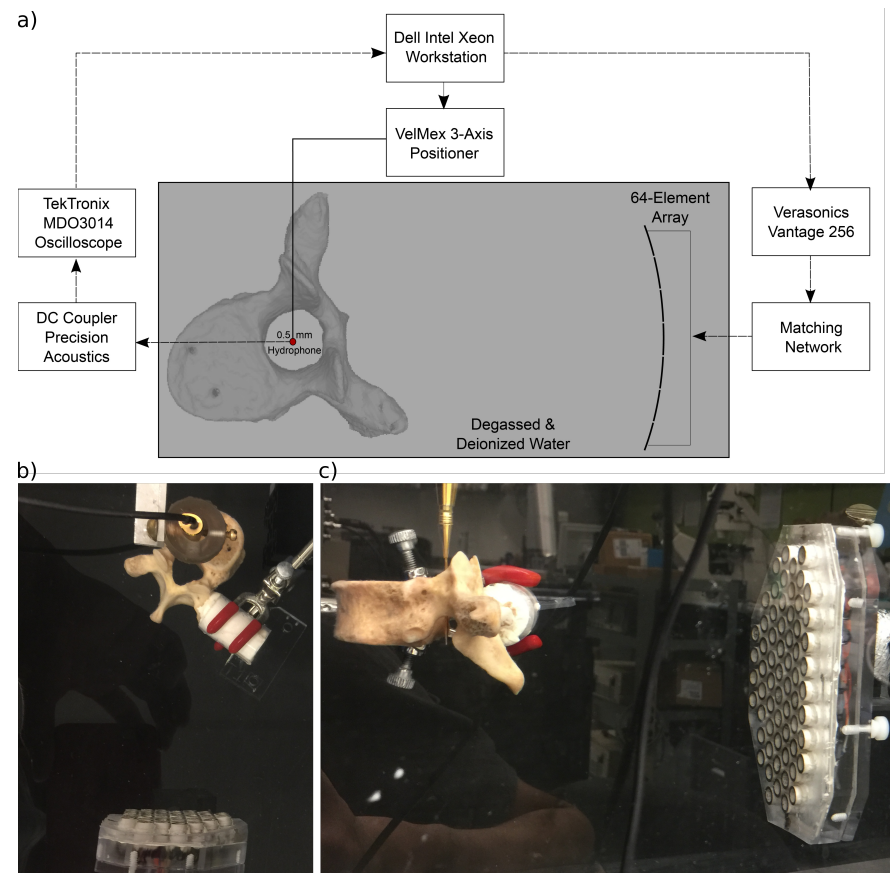

Fig. 1. a) Experimental setup, not to scale. The vertebra canal is approximately $12 \mathrm{~cm}$ from the b) overhead view and c) side view of an experiment.

The Velmex positioning system was used to translate the hydrophone to obtain the coordinates of five anatomical landmarks on the superior vertebra surfaces. The corresponding coordinates of the landmarks were obtained from the CT images of the vertebrae, and the affine transformation from CTspace to experimental vertebra-array configuration was applied to register the vertebra in simulation space. The positioning system was used to perform $10 \times 10 \mathrm{~mm}^{2}(0.25 \mathrm{~mm}$ step size $)$ raster scans in the $(x=0, y, z)$ plane in the vertebra canal to assess the focal distortions with and without vertebra-specific beamforming.

\section{Beamforming Methods}

Three transvertebral focusing methods were tested: 1) geometric delays, 2) hydrophone-based corrections generated by exciting one element at a time, recording the signal at the hydrophone, and calculating the time of flight through water and vertebra, and 3) CT-based ray acoustics corrections.
The delays generated from methods 2 and 3 were added to the delays from method 1 , which were obtained using signal transmission times from the individual elements to the hydrophone, propagating through water alone. Vertebrainduced delays were calculated retrospectively using k-Wave for comparison with the hydrophone and ray acoustics delays.

Hydrophone-based corrections were obtained by measuring the vertebra-induced delays for each element. The elements in the 64-element array were driven sequentially with a single-cycle pulse, recorded at the target with the hydrophone and digitized by the oscilloscope (1E+4 samples/waveform, 500E+6 sampling rate). Time delays for each element were measured by cross-correlating a $400 \mathrm{kHz}$ Gaussian pulse with a fractional bandwidth of 0.4 (generated with Matlab's gauspulse) with the waveforms recorded at the hydrophone.

Transvertebral ultrasound propagation was modelled using a multi-layered ray acoustics model [4]. The CT data of the vertebrae was segmented in ITK-SNAP using a semi-automatic segmentation algorithm [7]. Bone meshes were generated using binary masks of CT images of the vertebrae (0.503 x 0.503 $\mathrm{x} 0.5 \mathrm{~mm}$ discretization) using the open source ISO2MESH mesh generation Matlab package [8], and vertebral acoustic property maps were interpolated from frequency-densityacoustic property relationships measured in skull [9]. A mesh discretization study on the change in simulated vertebrainduced time delay was performed. The results are displayed in Fig. 2. Figure 2 shows that the change with an additional pointper-wavelength / mesh element per wavelength converges to below $0.02 \mu \mathrm{s}(<1 \%$ of a wavelength at $400 \mathrm{kHz})$ by the time the mean element size is $(\lambda / 12)^{2}$. The mesh discretization was set to $(\lambda / 12)^{2}$ accordingly.

Transvertebral ultrasound propagation was also modelled using the kspaceFirstorder3DG k-Wave code [6]. The $3 \mathrm{D}$ density, attenuation, and speed of sound maps generated from the vertebral CT data were linearly interpolated to the kWave grid. Compressional sound speed values were set using an optimized vertebra lamina specific linear density-sound speed model. The attenuation coefficients were converted from $\mathrm{Np} / \mathrm{m}$ (interpolated from [9] to $400 \mathrm{kHz}$ ) to $\mathrm{dB} / \mathrm{MHz} \cdot \mathrm{cm}$. The 64-cylindrical element array was created by using the $\mathrm{k}$ Wave toolbox makeBowl to generate a set of voxelized discs with $7.5 \mathrm{~mm}$ diameters (element outer diameter) and a set of discs with $5.3 \mathrm{~mm}$ diameters (element inner diameter), then taking the difference of the two masks. The modeled source signal was obtained from a signal measured in water with the needle hydrophone. The signals recorded at the receivers were averaged by element and the group time delay was extracted via cross-correlation of the input waveform and the received waveform. A spatial discretization convergence test on the vertebra-induced delays was performed. The k-Wave delays converge at a similar rate to the ray acoustics delays (Fig. 2) and the spatial discretization of the k-Wave model was set as $\lambda / 12$ accordingly ( $<1 \%$ of a wavelength change with increased discretization). The CFL-number was set to 0.05 throughout, giving a temporal discretization of $3.59 \mathrm{~ns}$ at 
$\lambda / 12$ spatial discretization. A 10 voxel perfectly matched layer was used. The computation time for a domain size of 486 by 192 by 432 grid points ( 151.00 by 59.65 by $134.22 \mathrm{~mm}$ ) was approximately 15 minutes using an NVIDIA RTX 2080 Ti.
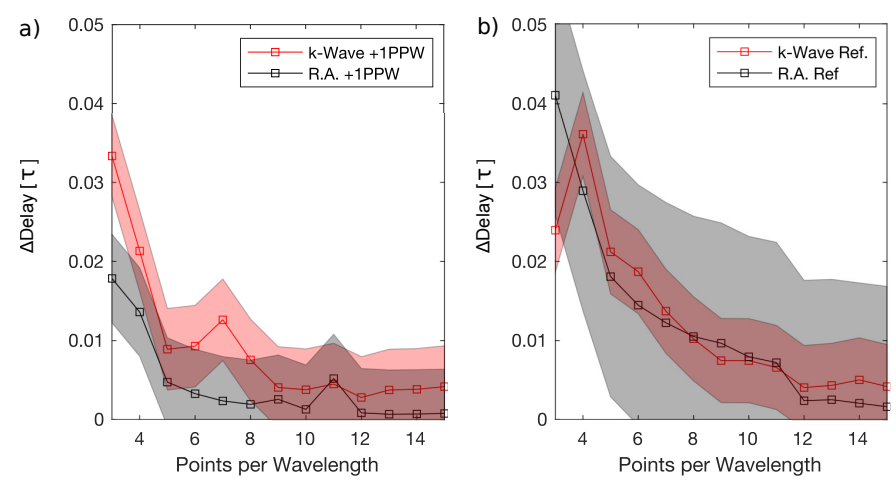

Fig. 2. The change in array mean and standard deviation in vertebra-induced delays when the discretization is increased by one point-per-wavelength $(+1 \mathrm{PPW})$ (a) or (b) relative to the delays calculated at 16 points-perwavelength.

\section{RESULTS}

\section{A. Array Focus in Water}

The hydrophone was positioned approximately $12 \mathrm{~cm}$ from the surface of the array, along the $\mathrm{x}$-axis. The array focus generated with the hydrophone delays in water (each element driven at $30 \mathrm{~V}$ ) are displayed in a 2-dimensional slice and along the three axes of the experimental system in Fig. 3
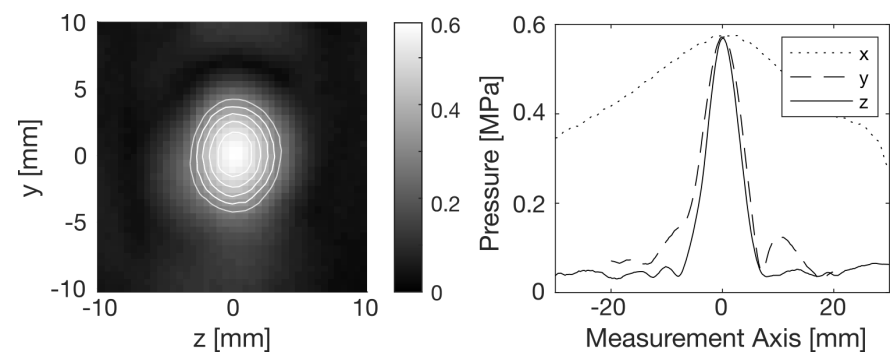

Fig. 3. a) 2D slice and b) $1 \mathrm{D}$ axial slices of the array pressure amplitude in water. The contours begin at $50 \%$ and increase in $10 \%$ increments.

The vertical and lateral full-width at half-maxima were $6.3 \mathrm{~mm}$ and $8.3 \mathrm{~mm}$, the latter being less than half the diameter of the vertebral canal. The elements produced a pressure amplitude of nearly $0.6 \mathrm{MPa}$ at the focus at the $30 \mathrm{~V}$ driving voltage. The elements may be driven safely with a single cycle excitation with a driving voltages up to $90 \mathrm{~V}$, resulting in a total peak negative pressure of close to $1 \mathrm{MPa}$. When combined with the laterally symmetric transvertebral component and the two paralaminar components, along with an assumed 30\% average transmission efficiency through the vertebral column [4, 5], the array should be capable of delivering at least $1 \mathrm{MPa}$ in situ, which is sufficient to induce BSCB-opening [10].

\section{B. Array Focus in Single Vertebral foramen}

In some cases, foci can be generated on target in the vertebral canal without incorporating aberration corrections. Figure 4 4 ) displays an example where the focus was generated on target using geometric delays. However, compensating for the time delays induced by the vertebra increases the focal pressure at the intended target (Fig. 4p). In this case, the increase in focal pressure when using vertebra-specific delays instead of geometric delays was $32 \%$.
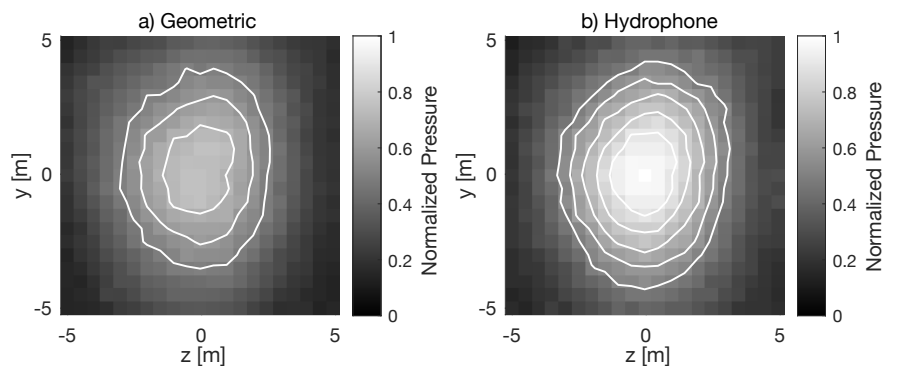

Fig. 4. YZ-slices of a focus generated in the T8 canal using a) geometric and b) hydrophone corrections. Pressures are normalized by the maximum pressure in b), and the contours range from $50 \%$ to $90 \%$ (10\% intervals).

A set of foci were generated with the spine-specific phased array in each of the individual thoracic vertebrae (T1, T2, ... T12). Figure 5 shows examples of aberrated foci from each of the thoracic vertebrae. Hydrophone corrections were then implemented using delays restricted to $(-\lambda, 0]$. Figure 5 shows several examples of foci (T3, T6, T8) that are vertically or laterally (T12) split using when the array geometrically focused to the canal, then reconstituted with hydrophone corrections. The average root-mean-square focal shift with geometric delays was $2.4 \pm 1.7 \mathrm{~mm}$, while the average root-meansquare focal shift with hydrophone delays was $1.3 \pm 1.1 \mathrm{~mm}$.

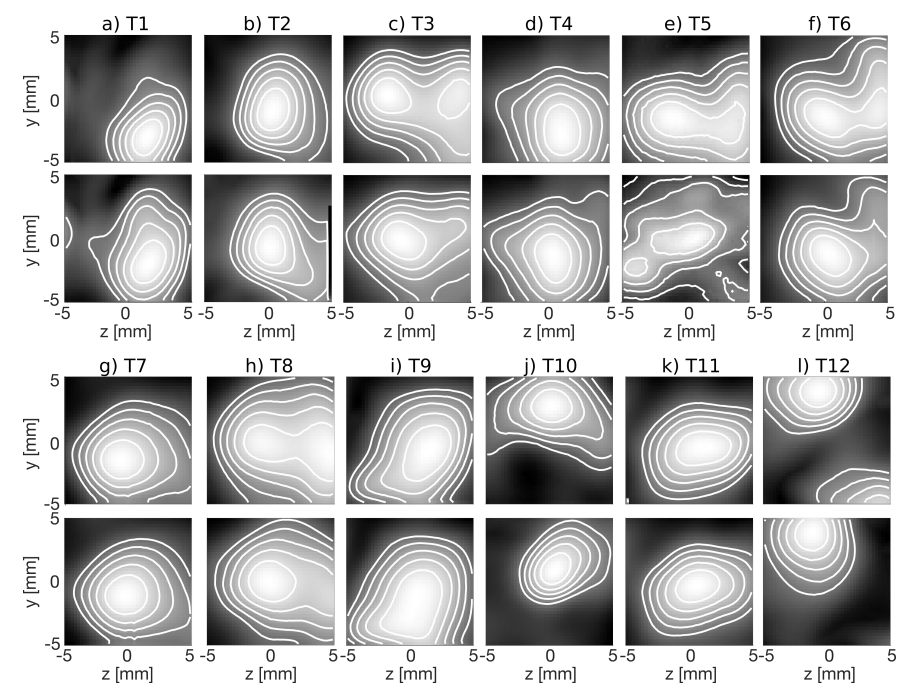

Fig. 5. Foci generated through the posterior arches of T1 to T12, generated with geometric focusing (top) and hydrophone focusing (bottom). Contours span $50 \%$ to $90 \%$ normalized pressure in $10 \%$ increments. 

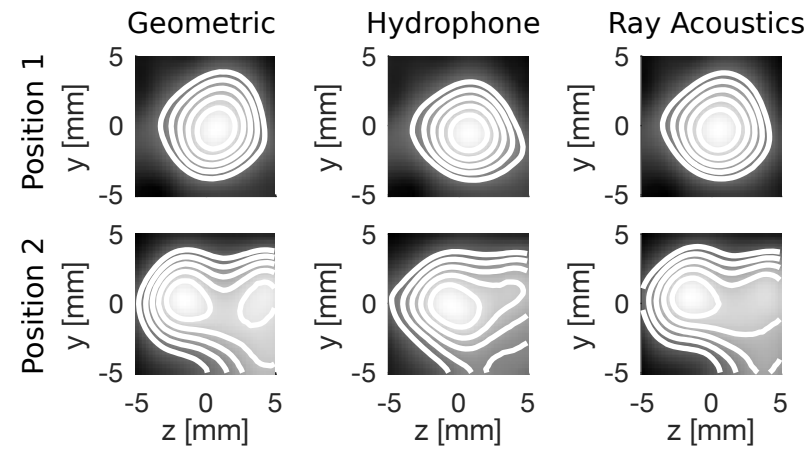

Fig. 6. Geometric, hydrophone, and ray acoustics foci for two T3 positions ( $2.5 \mathrm{~mm}$ vertical shift). Contours span $50 \%$ to $90 \%$ normalized pressure in $10 \%$ intervals.

\section{Foci Generated with Simulated Delays}

An experiment was performed with T3 and with three forms of aberration corrections; geometric delays, hydrophone delays, and ray acoustics delays. Figure 6 shows a subset of the experiments performed in T3; one example of a relatively undistorted focus (Position 1) and one example with a focus that is distorted (Position 2) when generated using geometric delays. There was no distinguishable difference between the three focusing methods in Position 1. However, Position 2 showed a split-focus pattern with geometric focusing. The split foci were reconstituted with the ray acoustics and hydrophone delays, although more convincingly with hydrophone delays.

\section{Delays: Hydrophone vs. Ray Acoustics vs. k-Wave}

The delays used to generate the foci in Fig. 6. T3, are displayed in Fig. 7 for each element in the 64-element array, along with the numbering scheme for the array elements. The accuracy of the models was quantified with the normalized correlation between the experimental and simulated delays, $\overline{s_{c}}$. The ray acoustics model had $\overline{s_{c}}=0.04$ for $\mathrm{T} 3$, position 1 , despite the delays for elements one to 30 appearing wellcorrelated, and $\overline{s_{c}}=0.71$ for $\mathrm{T} 3$, position 2 . The $\mathrm{k}$-Wave model had $\overline{s_{c}}=0.36$ for $\mathrm{T} 3$, position 1 , and $\overline{s_{c}}=0.69$ for T3, position 2; higher on average than those of the ray acoustics model.
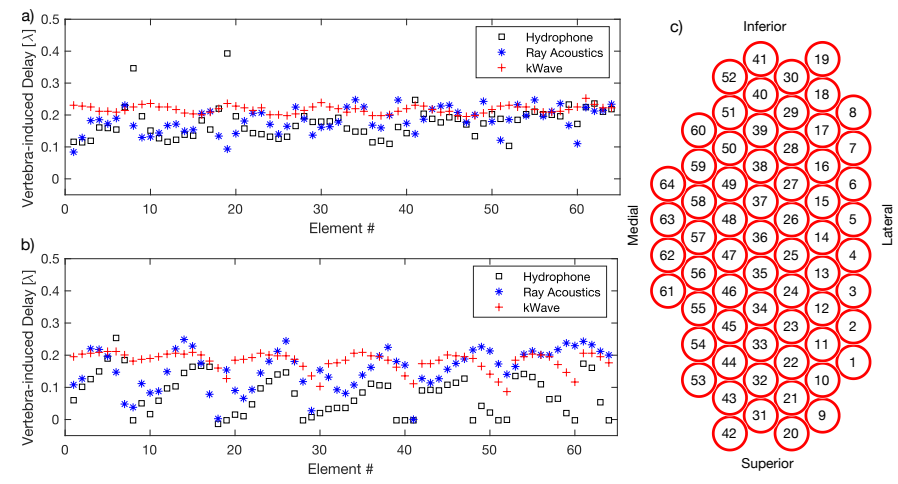

Fig. 7. The vertebra-induced delays for each of the array elements, for T3 a) position 1 and b) position 2. c) The array element numbering scheme.

\section{Conclusion}

In this work, we demonstrate the feasibility of transvertebral beamforming with a 64-element array modified from the design of a quadrant of a 256-element spine-specific array. We estimate that the full spine-specific array is capable of producing $1 \mathrm{MPa}$ in situ. The array was capable of producing foci in the vertebral canal using short pulses and geometric delays that were an average of $2.4 \pm 1.5 \mathrm{~mm}$ away from target. Hydrophone-based corrections were tested with delays restricted to $(-\lambda, 0]$, producing foci an average of $1.3 \pm 1.1 \mathrm{~mm}$ away from the target. The vertebra-induced delays were simulated with two methods; a multi-layered ray acoustics model and retroactively with the fluid k-Wave model; the delays calculated with the ray acoustics model demonstrated improved focal localization relative to the geometric delays.

\section{REFERENCES}

[1] Danielle Weber-Adrian et al. "Gene delivery to the spinal cord using MRI-guided focused ultrasound". In: Gene Ther. 22.7 (2015), pp. 568-577.

[2] Paige Smith et al. "Characterization of ultrasoundmediated delivery of trastuzumab to normal and pathologic spinal cord tissue". In: Sci. Rep. 11.1 (2021), pp. 1-12.

[3] Kullervo Hynynen and Ryan M Jones. "Image-guided ultrasound phased arrays are a disruptive technology for non-invasive therapy". In: Phys. Med. Biol. 61.17 (2016), R206.

[4] Rui Xu and Meaghan A O'Reilly. "Simulating transvertebral ultrasound propagation with a multi-layered ray acoustics model". In: Phys. Med. Biol. 63.14 (2018).

[5] Rui Xu and Meaghan A O'Reilly. "A spine-specific phased array for transvertebral ultrasound therapy: design \& simulation”. In: IEEE TBME (2019).

[6] Bradley E Treeby and Benjamin T Cox. "k-Wave: MATLAB toolbox for the simulation and reconstruction of photoacoustic wave fields". In: J. Biomed. Opt. 15.2 (2010), p. 021314.

[7] Paul A Yushkevich et al. "User-guided 3D active contour segmentation of anatomical structures: significantly improved efficiency and reliability". In: Neuroimage 31.3 (2006), pp. 1116-1128.

[8] Qianqian Fang and David A Boas. "Tetrahedral mesh generation from volumetric binary and grayscale images". In: ISBI. Ieee. 2009, pp. 1142-1145.

[9] Samuel Pichardo, Vivian W Sin, and Kullervo Hynynen. "Multi-frequency characterization of the speed of sound and attenuation coefficient for longitudinal transmission of freshly excised human skulls". In: Phys. Med. Biol. 56.1 (2010), pp. 219-250.

[10] Stecia-Marie P Fletcher et al. "A porcine model of transvertebral ultrasound and microbubble-mediated blood-spinal cord barrier opening". In: Theranostics 10.17 (2020), p. 7758. 